\title{
Erratum: Nonadiabatic dynamics of superfluid spin-orbit-coupled degenerate Fermi gas [Phys. Rev. A 92, 053626 (2015)]
}

\author{
Maxim Dzero, Ammar A. Kirmani, and Emil A. Yuzbashyan
}

(Received 16 July 2021; published 20 August 2021)

DOI: 10.1103/PhysRevA.104.029905

The steady-state (phase) diagram derived in our original paper (see original Fig. 2) is incorrect as a result of a mistake in the analysis of the isolated roots of the norm of the Lax vector. The correct phase diagram [1] appears in Fig. 1 here. The main difference is the absence of the island of phase II inside phase III. Note the alternative terminology-here, we refer to regions (regimes) I, II, and III as phases I, II, and III, respectively.

Further, the pseudospin equation of motion in our original paper [Eq. (2.7)] is missing a term. The correct equation [1] reads

$$
\dot{\mathbf{S}}_{\mathbf{k} \lambda}=\mathbf{B}_{k \lambda} \times \mathbf{S}_{\mathbf{k} \lambda}+\mathbf{m}_{k} \times \mathbf{L}_{\mathbf{k} \lambda}-\mathbf{m}_{k} T_{\mathbf{k}} .
$$

Equation (2.7) in our original paper is missing the last term on the right-hand side. This additional term, however, does not affect the dynamics qualitatively.

Among our conclusions, the statement that the spin-orbit interaction drastically reduces the size of phase III in the phase diagram is incorrect, as Fig. 1 shows. The rest of our main conclusions are not affected by the above errors.

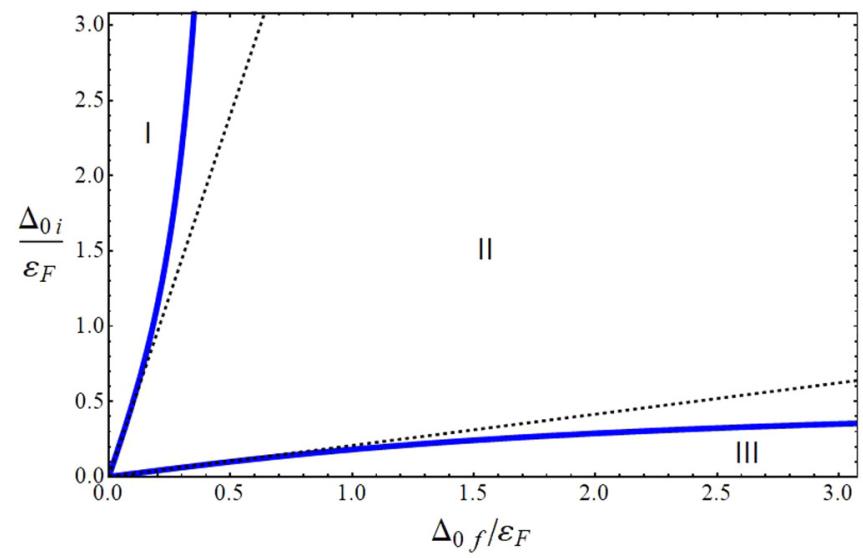

FIG. 1. Phase diagram for interaction quenches in the integrable limit $h_{Z}=0$ of the spin-orbit model. The black dotted lines $\Delta_{0 i}=$ $e^{ \pm \pi / 2} \Delta_{0 f}$ indicate the weak-coupling limit phase boundaries (see Ref. [1] for details). The thick blue lines mark the true phase boundaries. The notion of phases I, II, and III in this figure is the same as that of regions (or regimes) I, II, and III in the main text.

[1] J. A. Scaramazza, P. Smacchia, and E. A. Yuzbashyan, Consequences of integrability breaking in quench dynamics of pairing Hamiltonians, Phys. Rev. B 99, 054520 (2019). 